\title{
REV-ERB-erating nuclear receptor functions in circadian metabolism and physiology
}

\author{
Jürgen A Ripperger ${ }^{1}$, Urs Albrecht ${ }^{1}$ \\ ${ }^{I}$ Department of Biology, Unit of Biochemistry, University of Fribourg, Chemin du Musée 5, 1700 Fribourg, Switzerland \\ Cell Research (2012) 22:1319-1321. doi:10.1038/cr.2012.81; published online 22 May 2012
}

\begin{abstract}
A hallmark of the mammalian circadian timing system is synchronization of physiology and behavior, but when this synchronization is disturbed, chronic diseases such as metabolic syndrome and depression may develop. Three new studies show that nuclear receptors of the Rev-Erb family impact the circadian oscillator and its metabolic output and this can be modified with specific agonists. Hence, resynchronization of metabolic pathways by manipulation of the circadian oscillator using REVERB-specific agonists may represent a feasible therapeutic concept to target diseases rooted in a misaligned circadian system.

The circadian clock simulates the external light:dark cycle in essentially all body cells [1]. The coarse separation into activity and rest phase optimally synchronizes anabolic and catabolic pathways not only in individual cells, but also in the surrounding tissue and even the entire organism. A paired neuronal structure in the hypothalamus, the so-called Suprachiasmatic nuclei $(\mathrm{SCN})$, keep the organism in resonance with the environment. This master clock converts the external light:dark information into hormonal and electrophysiological signals synchronizing all
\end{abstract}

Correspondence: Urs Albrecht

E-mail: urs.albrecht@unifr.ch other cell-autonomous circadian clocks in the periphery. Like this stable phaserelationships between all of the organs are established. By definition ('circa diem' translates to 'about a day') circadian clocks provide a period length of about a day. However, this slight daily imprecision is not a problem, because sophisticated regulatory mechanisms, e.g., based on light input or food uptake, provide sufficient flexibility to the clock mechanism to readjust every day to the environment.

Many diseases may be related to disturbances of specific metabolic or physiological pathways. For instance, in metabolic syndrome the balance between the energy-generating glucose metabolism and the energy-storing lipid metabolism is disturbed [2]. On the other hand, de-synchronization of hormone secretion and clearance aggravates neuropsychiatric disorders such as seasonal affected disorder (SAD), depression or bipolar disorder [3]. The finding that strict daily routines of sleeping and eating times, or light therapy can ameliorate to a certain degree these neuropsychiatric disorders [4] highlights the importance of the circadian system to maintain the normal state. Hence, drugs that readjust the synchronizing properties of the circadian clock would be beneficial. For example, lithium is used to treat bipolar disorder and impacts various molecular pathways including components of the circadian clock [5].

At the heart of the circadian clock is a molecular oscillator based on transcriptional and post-translational feedback loops [1]. Briefly, a heterodimer composed of BMAL and CLOCK (or NPAS2 in the brain) activates transcription of two sets of repressors (blue line; Figure 1). The first set (i.e., Per and Cry) feeds back via E-box motifs (black boxes) after a certain delay on its own synthesis (pink lines) and these consecutive cycles of activation and repression create transcriptional rhythms of about 24 $\mathrm{h}$. The second set was thought to solely regulate rhythmic accumulation of the Bmal1, Clock and Npas2 genes (red lines) via RORE motifs (white boxes). This second, stabilizing feedback loop relies on alternate repression by REV$\mathrm{ERB} \alpha$ and activation by ROR $\alpha$ (not shown). Input factors such as light (via induction of Per genes) or food uptake (via metabolism) affect the phase of the system (grey lines). Circadian output target genes regulating for example key enzymes of metabolic pathways can be either directly coupled to the molecular oscillator via E-box and/or RORE motifs, or via rhythmically expressed transcription factors as intermediates. Among the different families of transcriptional regulators, members of the family of nuclear receptors link the circadian timing system with metabolism $[6,7]$. In this fashion, tissue-specific intertwined networks of gene expres- 


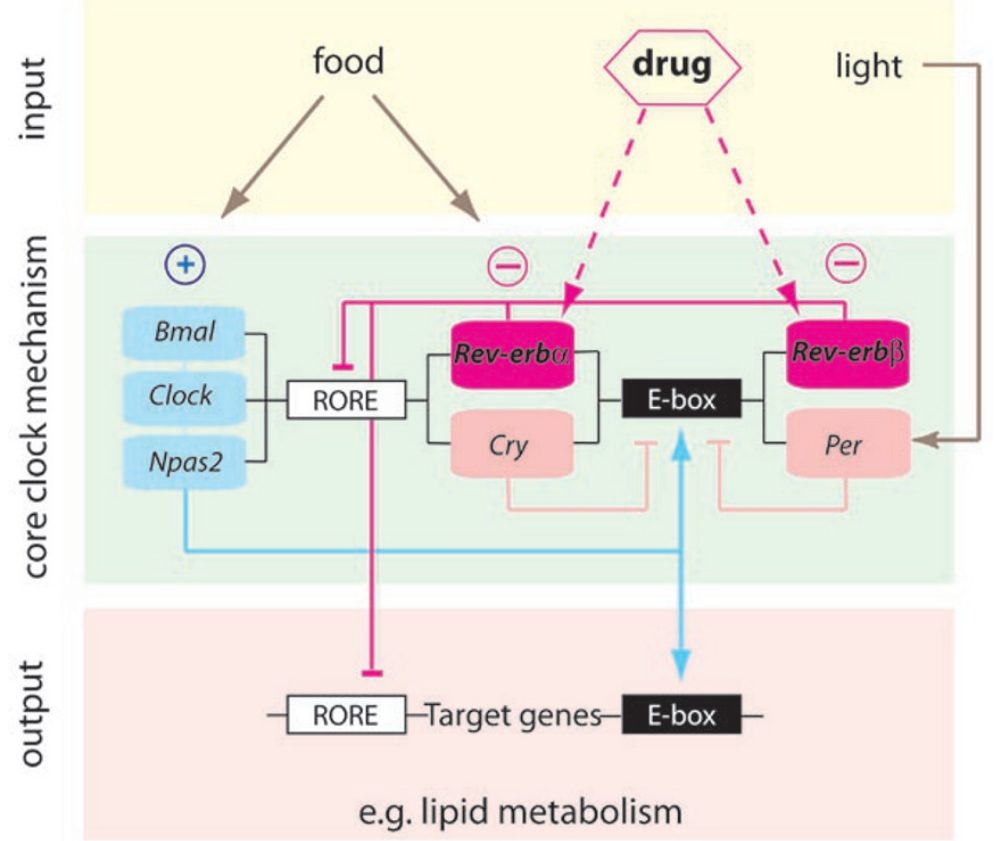

Figure 1 Impact of nuclear receptors of the Rev-Erb family on the circadian oscillator and their effect on metabolism. Blue: positive factors; red, pink: two sets of negative factors. The core clock mechanism governs rhythmic expression of output genes. The phase of the system is every day readjusted by light or food uptake (input; grey arrows). Specific agonists for nuclear receptors of the RevErb family mimic these adjusting properties and may impact on metabolic functions. For details, see text.

sion are generated. This is the base for the temporal synchronization of the multiple metabolic pathways.

Experiments by Bugge et al. [8] and Cho et al. [9] combining chromatin-immunoprecipitation (ChIP)-sequencing with powerful knockout animal systems shed new light on the function of both nuclear receptors of the Rev-Erb family within the circadian transcriptional network. Surprisingly, many new RORE motifs were identified in core circadian oscillator genes (Figure 1) and transcriptome analysis of Rev-Erb-deficient mice revealed that the expression of these genes was actually affected. Hence, the REV-ERB nuclear receptors have a more prominent function within the circadian oscillator than previously thought, which became apparent only after the inactivation of both Rev-Erba and $\beta$ isoforms. Mice lacking both isoforms displayed a shorter circadian rhythm, which resembles mice deficient in other circadian oscillator components. Interestingly, the lipid metabolism, and to some extent cholesterol and bile acid production, were severely affected, with the liver displaying a typical lipid overstorage phenotype, i.e., hepatosteatosis. As conclusion, the balance of lipid and glucose metabolism is disturbed in these animals. Considering the fact that these nuclear receptors impact circadian oscillator and output functions and that at least $R E V-E R B \alpha$ readjusts its phase in response to external influences on the circadian timing system [10], targeted activation of these factors using agonists may improve coordination of metabolic pathways.

Solt et al. [11] now identified a new class of agonists for nuclear receptors of the Rev-Erb family. In vitro, they bound specifically and with high affinity, and suppressed circadian reporter genes in cultured cells or SCN explants. In vivo, administration of these drugs at the peak of REV-ERB activity suppressed circadian locomotor activity and REVERB target gene expression. Hence, these drugs overcame the blood-brain barrier to exert their function even in the SCN. Surprisingly, the activity of these drugs was stronger in the absence of light but it was not determined whether this was due to a faster clearance of these drugs from the circulation in the presence of light, or due to competition of their action with the light input pathway because they mimic input function (Figure 1). In addition, these drugs increased fatty acid and glucose oxidation, while reducing the amount of lipogenesis and bile acid synthesis. As a consequence, treated mice became leaner and were more resistant to the detrimental effects of high-fat diet. Interestingly, these drugs reduced weight gain in obese mice that lack leptin, indicating that the newly discovered REV-ERB agonists affect at least partially metabolic pathways downstream or independent of leptin. Taken together, specific agonists for nuclear receptors of the REV-ERB family hold the potential to resynchronize metabolic pathways in living organisms. Potential applications concern metabolic syndrome, Jet-lag (i.e., desynchronization of SCN and peripheral clocks), and possibly neuropsychiatric disorders. Future studies will have to address the effect of these drugs on the central nervous system, especially whether and how sleep- and mood-related behaviors are affected. It will be challenging and exciting to translate basic research performed in mice to the clinic for treatment of patients.

\section{References}

1 Huang W, Ramsey KM, Marcheva B, Bass J. Circadian rhythms, sleep, and metabolism. J Clin Invest 2011; 121:2133-2141. 
2 Levinson RS. Metabolic syndrome. Nat Med 2011; 17(Suppl):4-7.

3 Ciarleglio CM, Resuehr HE, McMahon DG. Interactions of the serotonin and circadian systems: nature and nurture in rhythms and blues. Neuroscience 2011; 197:8-16.

4 Hlastala SA, Frank E. Adapting interpersonal and social rhythm therapy to the developmental needs of adolescents with bipolar disorder. Dev Psychopathol 2006; 18:1267-1288.

5. Li J, Lu WQ, Beesley S, Loudon AS, Meng QJ. Lithium impacts on the amplitude and period of the molecular circadian clockwork. PLoS One 2012; 7:e33292

6 Schmutz I, Ripperger JA, BaeriswylAebischer S, Albrecht U. The mammalian clock component PERIOD2 coordinates circadian output by interaction with nuclear receptors. Genes Dev 2010; 24:345-357.

7 Lamia KA, Papp SJ, Yu RT, et al. Cryptochromes mediate rhythmic repression of the glucocorticoid receptor. Nature 2011; 480:552-556.

8 Bugge A, Feng D, Everett LJ, et al. Rev-erb $\alpha$ and Rev-erb $\beta$ coordinately protect the circadian clock and normal metabolic function. Genes Dev 2012; 26:657-667.
9 Cho H, Zhao X, Hatori M, et al. Regulation of circadian behaviour and metabolism by REV-ERB- $\alpha$ and REVERB- $\beta$. Nature 2012; 485:123-127.

10 Stratmann M, Stadler F, Tamanini F, van der Horst GT, Ripperger JA. Flexible phase adjustment of circadian albumin D site-binding protein (DBP) gene expression by CRYPTOCHROME1. Genes Dev 2010; 24:1317-1328.

11 Solt LA, Wang Y, Banerjee S, et al. Regulation of circadian behaviour and metabolism by synthetic REV-ERB agonists. Nature 2012; 485:62-68. 